\title{
Toma de decisiones en menores con factores de riesgo para el Trastorno Disocial de la Conducta.
} Jasmín Bonilla-Santos ${ }^{1}$, jasmin.bonillas@campusucc.edu.co, González-Hernández Alfredis ${ }^{2}$, Bonilla-Santos Gisella ${ }^{3}$, Muñoz-Bernal Luisa
Fernanda $^{4}$, Baldovino-Pérez Irma Elena ${ }^{5}$

Psicóloga. PhD. Neurociencias Cognitivas. Universidad Cooperativa de Colombia; Grupo Investigación Psicosaberes; Neiva, Colombia Psicólogo. PhD. Neurociencias cognitivas.Universidad Surcolombiana; Área bases biológicas del comportamiento humano; Neiva, Colombia Psicóloga. Universidad Surcolombiana; Grupo Investigación Dneuropsy; Neiva, Colombia,

Psicóloga. Especialista en Trastornos Afectivo y Emocionales. Universidad Surcolombiana; Grupo Investigación Dneuropsy; Neiva, Colombia Psicóloga, Maestrando Psicología Clínica. Docente investigadora Universidad Cooperativa de Colombia; Neiva, Colombia

Fecha de recepción Enero 15 de 2016 Fecha de correcciones Junio 12 de 2016 Fecha de aceptación Septiembre 30 de 2016

Fecha de publicación Enero 31 de 2017

\section{Resumen}

Introducción. Existe la hipótesis de que las emociones participan del proceso cognitivo de la toma de decisiones guiando la conducta y dotando a la experiencia de una cualidad positiva o negativa que permite la adaptación del individuo al medio social. Método: La muestra estuvo conformada por 81 menores de edad (48 que cumplían con uno o dos criterios del DSM-IV y 33 sin alteraciones de comportamiento). Se utilizó la Escala Muldimensional de la Conducta, el SNAP IV y el Check list para TDC como instrumentos de tamizaje para su selección, posteriormente se realizó la evaluación con la versión computarizada de la IGT (Iowa Gambling Task). Resultados: Se dan diferencias estadísticamente significativas entre el grupo estudio y de comparación para la selección de las barajas desventajosas B durante la ejecución de la tarea. Los menores en riesgo presentaron un desempeño menor para la prueba general de toma de decisiones. Discusión: Los resultados indican la presencia de alteraciones en los procesos de toma de decisiones de los menores que presentan uno o dos criterios clínicos para desarrollar el TDC.

Palabras claves: Toma de Decisiones, Trastorno Disocial, Gambling Task, Corteza Ventromedial.

Copyright $($ C Facultad de Ciencias de la Salud de la Universidad Tecnológica de Pereira. 1995-2017. Todos los derechos reservados *
Decision making in minors with risk factor of dissocial behavior disorder.

\section{Abstract}

Introduction: There is a hypothesis that proposes that emotions take part in the cognitive process related to decision-making behavior; such processes provide experience of a positive or negative quality that allows the adaptation of a human being to a social background. Methods: There were 81 minors participants (48 qualified with one or two standards of DSM-IV and 33 without conduct disorders). We used Multidimensional Scale of Conduct, SNAP IV, and checklist for $\mathrm{CD}$ as screening instruments for its selection, and later we performed the assessment with the online version of IGT (Iowa Gambling Task). Results: A statistically significant difference was present between study and control groups in the selection of unfavorable decks of cards. Those children affected by behavioral disorders presented lower results in the general decision-making test. Conclusions: Results suggest the presence of disorders in decision-making in minors that present one or two clinical criteria to develop CD. This could be due to difficulties in contingency processing for unfavorable decisions, which prevented them from generating somatic states based on the possible future consequences.

Keywords: Decision-making, conduct disorder, gambling task, ventromedial cortex.

Copyright ( $\odot$ Facultad de Ciencias de la Salud de la Universidad Tecnológica de Pereira. 1995-2017. All rights reserved ${ }^{\circ}$.

\section{Introducción}

La toma de decisiones se define como la habilidad para seleccionar el plan de acción de mayor adaptación para el organismo entre un conjunto de posibles alternativas conductuales [1], siendo un proceso que integra las funciones ejecutivas, referidas como la capacidad de satisfacer los impulsos y emociones básicas, acorde con las demandas ambientales y sociales $[2,3]$

En la toma de decisiones interactúan factores cognitivos y emocionales como el procesamiento de los estímulos presentes en la tarea, el recuerdo de experiencias pasadas, las emociones generadas y la valoración de las posibles consecuencias entre las diferentes opciones; sucesos que se promueven en el individuo mediatizados por otros procesos cognitivos como la memoria de trabajo, los sistemas atencionales y las funciones ejecutivas $[4,5]$. El procesamiento emocional es el resultado de las experiencias de sucesos similares propios o vicarios y los relacionados a las consecuencias o al contexto, éste antecede al componente cognitivo y actúa como una marca somática guiando la toma de decisiones [6].

Estos procesos cognitivos y emocionales que emergen de la toma de decisiones se relacionan especialmente con el funcionamiento de las áreas orbitofrontal y ventromedial (4,7-10), es allí donde se valoran las consecuencias a largo plazo de las decisiones que se eligen, como resultado de la integración de los estados somáticos con información relevante procedente de la propia situación o de la memoria a largo plazo producto de vivencias significativas $[6,11,12]$.

Alteraciones en estas áreas conllevan a la incapacidad para planificar, inflexibilidad atencional, dificultad en la identificación de emociones e inclinación por conductas de riesgo y agresivas [1, 11-13]; los hallazgos en las investigaciones refieren la importancia de estudiar la toma de decisiones durante la infancia, por ser en esta etapa donde se empiezan a generar los comportamientos de riesgo los cuales se acentúan en la adolescencia como toma de decisiones desventajosa y perjudicial hacia el futuro [14].

La toma de decisiones desventajosa puede llegar a ser patológica y conducir a problemas complejos [12], como los asociados al consumo de sustancias psicoactivas [15-17]; el comportamiento suicida [18, 19] y los trastornos alimenticios [20, 21], de igual forma los comportamientos disruptivos y violentos de los menores y adolescentes ha sido de interés investigativo [14,22, 23], debido a que constituyen uno de los problemas de mayor relevancia en el ámbito escolar y se han convertido en el principal motivo de consulta clínica de los padres.

El presente estudio plantea la hipótesis de las selecciones de riesgo en niños y niñas como un factor predictor para el desarrollo de trastornos comportamentales y se propone como objetivo evaluar la toma de decisiones mediante el desempeño en la tarea IGT en niños y niñas que cumplían 
con uno o dos criterios según el DSM-IV (24) para desarrollar el TDC.

\section{Materiales y Método}

Estudio transversal con diseño descriptivo comparativo; el muestreo fue de tipo intencional teniendo en cuenta los criterios de inclusión: 40 niños y 8 niñas vinculados a escuelas públicas con edades comprendidas entre los 9 a 14 años, que presentaban uno o dos de los criterios señalados en el DSM-IV (24) para el diagnóstico de TDC y de quienes se obtuvo el consentimiento informado de los padres o acudientes para participar en la investigación, comparados con un grupo de 31 niños y 2 niñas con características sociodemográficas similares pero sin criterios para TDC.

Para los dos grupos se tuvo en cuenta los siguientes criterios de exclusión: consumo de sustancias psicoactivas, diagnósticos de trastornos comportamentales (Trastorno Déficit de Atención e Hiperactividad, Trastorno Oposicionista Desafiante, Trastorno Disocial de la Conducta de inicio infantil), resultado inferior a 70 puntos en la escala WISC, alteraciones neuropsicológicas de base e inicio de conductas delictivas.

El protocolo de investigación y el consentimiento informado fueron aprobados por el Comité de Ética en Investigación de la Facultad de Salud de la Universidad Surcolombiana.

\section{Instrumentos de Tamizaje}

Lista de síntomas para el TDC (Check list), cuestionario conformado por 14 síntomas del criterio diagnóstico A del DSM-IV (24), se manejó la versión padres, docentes y autoinforme, con el fin de determinar la ausencia de este trastorno en la muestra seleccionada para el estudio de acuerdo con los criterios de inclusión.

Escala Multidimensional de la Conducta (EMC): versión estandarizada y validada en Colombia [25]. Este sistema de evaluación proporciona cuestionarios validados para la identificación característica de la conducta y de la personalidad relacionada con diferentes nosologías diagnósticas del DSM-IV de niños y adolescentes de 4 a 18 años. Incluye dimensiones positivas (adaptativas) y negativas (clínicas), donde se establece la severidad de los síntomas y un diagnóstico diferencial.

Escala de medición del comportamiento del Trastorno por Déficit de Atención e Hiperactividad (SNAP), versión padres y docentes (26), se empleó para la identificación del TDAH de acuerdo con los criterios de inclusión.

\section{Instrumentos de Evaluación}

La IGT es una tarea de toma de decisiones que parte de los supuestos establecidos en la Teoría de los Marcadores Somáticos TMS para situar al sujeto en la necesidad de elegir entre cuatro opciones (cuatro barajas de cartas), busca reflejar condiciones similares de la vida cotidiana en la toma de decisiones; implica la evaluación anticipada $\mathrm{y}$ de forma intuitiva de las consecuencias futuras relacionadas con las acciones realizadas [27].

Se utilizó una versión computarizada, basada en la tarea original del IGT, consiste en la observación del participante hacia la pantalla del ordenador encontrando cuatro barajas de cartas iguales en apariencia y tamaño (con las etiquetas A', B', C', y D' y ubicadas en la parte inferior de cada baraja). El participante debe escoger libremente cartas de las cuatro barajas con el mouse, las cuales le permitirán ir ganando o perdiendo algo de dinero (puntos) durante el desarrollo de la tarea. Cada baraja tiene 60 cartas, y la tarea consta de un total de 100 ensayos, por lo que es posible (aunque no frecuente) que el sujeto agote todas las cartas de determinada baraja. En la parte superior derecha de la pantalla del ordenador se ubica una barra roja que indica el crédito con el que cuenta el participante para el desarrollo del juego (inicialmente será de 2000 dólares), también se encontrará una barra verde que irá aumentando o disminuyendo a medida que vaya ganando o perdiendo dinero. La tarea se acompaña de imágenes con una cara alegre o triste y sonidos característicos dependiendo si ha obtenido una ganancia o pérdida. El participante no tiene ningún indicio previo sobre el contenido de las cartas, por lo que debe ir identificando cuales son las cartas buenas (asociadas con ganancia) y cuales las malas (asociadas con pérdida) a lo largo del juego. [11, 27].

Lo característico de la tarea de apuesta IGT radica en que algunas barajas son más favorables que otras. Al seleccionar alguna carta de las barajas A y B proporcionan altas ganancias (100 puntos), sin embargo los castigos también son altos. En la baraja A la frecuencia de los castigos aumenta progresivamente, pero se mantiene constante la magnitud del castigo (pérdida de puntos); en la baraja B la frecuencia de los castigos se mantiene constante, pero la cantidad en la pérdida de puntos aumenta relativamente.

En las barajas C y D las ganancias son más bajas (50 puntos), pero los castigos son más pequeños. En la baraja $C$ la frecuencia de penalización varía y se mantiene la magnitud del castigo, en la D la frecuencia de castigo es constante, pero la magnitud de los castigos se incrementa. Estas barajas son ventajosas porque permiten ganancia a largo plazo, mientras que las A y B son desventajosas porque proporcionan ganancias inmediatas pero pérdidas futuras [11, 28]

\section{Análisis Estadístico}

Se realizaron análisis descriptivos de la ejecución de los participantes en los ensayos de la IGT, con el fin de identificar las puntuaciones totales de la tarea. Se realizó un análisis estadístico mediante la prueba U Mann-Whitney, con el fin de identificar a cuál de las barajas se inclinaban los niños de ambos grupos. El análisis de los datos recogidos se realizó con el programa estadístico SPSS v. 15.0.

\section{Resultados}

La muestra objeto se distribuyó en dos grupos: el grupo de estudio 40 niños y 8 niñas quienes presentaban uno o dos criterios para el diagnóstico de TDC y el grupo de comparación de 31 niños y 2 niñas sin manifestaciones de alteraciones comportamentales, con edades comprendidas entre los 7 a 14 años y escolaridad de segundo a sexto grado.

La tabla 1 indica que los estudiantes de los dos grupos presentaron homogeneidad en las variables de edad y género, aunque se presentó una diferencia significativa en escolaridad a favor del grupo de estudio, se observó que fue este quien tuvo mayores elecciones para la baraja de cartas desventajosa (B.)

Tabla 1. Escolaridad y edad de los grupos

\begin{tabular}{|c|c|c|c|c|c|c|c|c|}
\hline \multirow{4}{*}{ Escolaridad } & \multirow{2}{*}{ Grupos } & \multicolumn{4}{|c|}{ Descriptivos } & \multicolumn{2}{|c|}{ Percentiles } & \multirow{2}{*}{ Valor $\mathrm{p}=$} \\
\hline & & Mediana & Mínimo & Máximo & Intercuartil & 25 & 75 & \\
\hline & Estudio & 5 & 3 & 6 & 1 & 4 & 5 & \multirow{2}{*}{0} \\
\hline & Comparación & 4 & 2 & 5 & 2 & 3 & 5 & \\
\hline \multirow{4}{*}{ Edad } & & \multicolumn{4}{|c|}{ Descriptivos } & \multicolumn{2}{|c|}{ Percentiles } & \multirow{2}{*}{ Valor $\mathrm{p}=$} \\
\hline & & Mediana & Mínimo & Máximo & Intercuartil & 25 & 75 & \\
\hline & Estudio & 11 & 9 & 14 & 1 & 10 & 11 & \multirow{2}{*}{0,07} \\
\hline & Comparación & 10 & 7 & 12 & 2 & 9 & 11 & \\
\hline
\end{tabular}


La tabla 2 muestra diferencias estadísticamente significativas en la selección de cartas de la baraja desventajosa B, siendo el grupo de estudio quien realizó con mayor frecuencia esta selección, lo cual se puede explicar probablemente por la magnitud de los pagos recibidos generando gratificación inmediata, activación de circuitos emocionales o circuito de recompensa y la poca reflexión de riesgo- beneficio de los castigos posteriores, circuitos cognitivos (14), aspectos que se relacionan con la predisposición que manifiestan los niños con TDC de presentar dificultades en el control inhibitorio, el análisis, la síntesis y la abstracción, emitiendo conductas arriesgadas basada exclusivamente en la carga emotiva (29, 30) mostrando cierta insensibilidad a los castigos recibidos y dificultad en el proceso de la retroalimentación adecuada frente a la toma de decisiones.

Tabla 2. Selección de barajas A-D

\begin{tabular}{|c|c|c|c|c|c|c|c|c|}
\hline \multirow{2}{*}{ Barajas } & \multirow{2}{*}{ Grupos } & \multicolumn{4}{|c|}{ Descriptivos } & \multicolumn{2}{|c|}{ Percentiles } & \multirow{2}{*}{ Valor $\mathrm{p}=$} \\
\hline & & Mediana & Mínimo & Máximo & Intercuartil & 25 & 75 & \\
\hline \multirow{2}{*}{ IGT-A } & Estudio & 22,5 & 10 & 29 & 4 & 21 & 24,75 & \multirow{2}{*}{0,088} \\
\hline & Comparación & 25 & 10 & 45 & 6 & 21 & 27 & \\
\hline \multirow{2}{*}{ IGT-B } & Estudio & 28 & 19 & 57 & 5 & 26 & 30,75 & \multirow{2}{*}{0,019} \\
\hline & Comparación & 26 & 21 & 39 & 4 & 24 & 28 & \\
\hline \multirow{2}{*}{ IGT-C } & Estudio & 25,5 & 17 & 37 & 6 & 22 & 28 & \multirow{2}{*}{0,787} \\
\hline & Comparación & 26 & 11 & 37 & 4 & 23 & 27 & \\
\hline \multirow{2}{*}{ IGT-D } & Estudio & 22 & 11 & 42 & 7 & 20 & 27 & \multirow{2}{*}{0,145} \\
\hline & Comparación & 25 & 12 & 37 & 4 & 22 & 26 & \\
\hline
\end{tabular}

Las selecciones para las barajas de las cartas ventajosas C y D no evidenciaron diferencias significativas entre los grupos, pero se observó una mayor frecuencia de selección para este tipo de cartas por parte del grupo de comparación.

\section{Discusión}

El objetivo del presente estudio fue evaluar el proceso de toma de decisiones en niños y niñas que cumplían con uno o dos criterios según el DSM-IV (24) para desarrollar el TDC, es decir que se encontraban en riesgo, comparados con un grupo de niños y niñas con comportamientos prosociales. Los resultados mostraron que los niños y niñas en riesgo presentaron un menor rendimiento en la toma de decisiones comparado con el grupo de pares con comportamientos socialmente adecuado, lo anterior se evidenció en las elecciones de cartas desventajosas de la baraja B.

Durante la ejecución de la tarea IGT, los participantes en riesgo para TDC realizaron menos selecciones de las cartas ventajosas, inclinándose con mayor frecuencia por las barajas que más puntos les suministraban de forma inmediata pero que a su vez presentaban un castigo mayor, por el contrario los participantes que no presentaban alteraciones a nivel comportamental mostraron una mayor tendencia a seleccionar las barajas $\mathrm{C}$ y D que le significaban menor recompensa inmediata pero ganancias futuras, eligiendo cada vez un número menor de cartas desventajosas durante la tarea.

Así, se observó que el grupo de comparación logró un aprendizaje en cuanto identificaron los niveles de castigo y recompensa de cada baraja, es decir que pudieron anticipar las consecuencias prospectivas de sus decisiones [1]; a diferencia del grupo de estudio que presentó un desempeño de incertidumbre durante la prueba mostrando poca sensibilidad al castigo, interfiriendo en la evaluación y dificultando el procesamiento del feedback y aprendizaje durante la tarea.

La IGT es una tarea de toma de decisiones que parte de los postulados establecidos en la Teoría de los Marcadores Somáticos (TMS) [31], la cual refiere que las respuestas viscerales aprendidas por asociación en experiencias pasadas (marcadores somáticos) son mecanismos que permiten sesgar las decisiones, al reducir la probabilidad de elegir determinadas opciones que han sido asociadas a un marcador negativo. Es así como las emociones participan del proceso cognitivo de la toma de decisiones guiando la conducta y dotando a la experiencia de una cualidad positiva o negativa que permite la adaptación del individuo al medio social [10, 32].

Los resultados demuestran la dificultad en el grupo de estudio para generar señales emocionales relacionadas a las pérdidas, que permitan un aprendizaje y reorientar las respuestas prospectivas en búsqueda de selecciones que remplacen las pérdidas mayores por ganancias futuras, por lo tanto sus decisiones negativas se fijaron en las barajas desventajosas; lo contrario ocurrió con el grupo de comparación quienes pudieron modificar su marcador de decisiones de acuerdo con las contingencias de cada una de las barajas, decidiendo al final por las de mayor ganancia a largo plazo.

La disfunción en el proceso de la toma de decisiones antecede a las manifestaciones clínicas y conductuales del TDC, esta investigación corrobora la hipótesis de la no generación del marcador somático en los niños y niñas que después de reunir algunos criterios clínicos desarrollan el trastorno. Se ha demostrado en el caso de adultos con lesiones a nivel orbitofrontal una tendencia hacia la selección fija de decisiones desventajosas, determinadas por la incapacidad para cambiar su patrón de elección[11], por lo cual perseveran de forma constante en las decisiones que son reforzadas inicialmente, situación evidenciada en el desempeño de la tarea IGT del grupo estudio, presentándose así una posible alteración en las áreas implicadas en el proceso de toma de decisiones también en esta población.

Hacia el futuro se crea la necesidad de realizar estudios que evalúen el proceso cognitivo de la toma de decisiones en muestras más amplias clasificadas por grupos etarios, que además de integrar el componente ejecutivo característico del TDC, incluya la valoración de los procesos relacionados con la cognición social y la medición de correlatos neuroanatómicos a partir de registros neurofisiológicos, para visualizar la activación cerebral de áreas específicas involucradas en los procesos valorados en el estudio, lo cual permitiría una identificación específica e integral de los predictores clínicos y comportamentales del TDC.

\section{Conflicto de interés}

Los autores manifiestan no tener ningún conflicto de interés

\section{Agradecimientos}

Este artículo es resultado de la investigación denominada "Intervención neurocognitiva componente "Teoría de la Mente" en niños escolares con Trastornos Comportamentales" con código 112456935069 financiada por el Departamento Administrativo de Ciencia, Tecnología e Innovación (COLCIENCIAS), Universidad Surcolombiana y Universidad Cooperativa de Colombia en la convocatoria 569 del año 2012 (Banco de Proyectos Elegibles de CTeI), mediante contrato número RC. No. 0202 - 2013 y desarrollada por los grupos de Investigación Dneuropsy de la Universidad Surcolombiana y Psicosaberes de la Universidad Cooperativa de Colombia sede Neiva 


\section{Referencias}

1. Bechara A, Damasio H, Damasio AR. Emotion, decision making and the orbitofrontal cortex. Cerebral cortex. 2000;10(3):295-307.

2. Guallart-Balet M, Jiménez-Cortés MP, Tuquet-Calvo H, PelegrínValero C, Olivera-Pueyo J, Benabarre-Ciria S, et al. Validación española de la Iowa Rating Scale for Personality Change (IRSPC) para la valoración de los cambios de personalidad en pacientes con daño cerebral adquirido. Revista de Neurología. 2015;17:29.

3. Ardila A. On the evolutionary origins of executive functions. Brain and cognition. 2008;68(1):92-9.

4. Martínez-Selva JM, Sánchez-Navarro JP, Bechara A, Román F. Mecanismos cerebrales de la toma de decisiones. Revista de neurología. 2006;42(7):411-8.

5. Barrutieta LH. Influencia de la emoción en la toma de decisión: implicación en la producción de inteligencia. Inteligencia y seguridad: Revista de análisis y prospectiva. 2011(10):103-22.

6. Bechara A, Damasio H, Damasio AR, Lee GP. Different contributions of the human amygdala and ventromedial prefrontal cortex to decision-making. The Journal of Neuroscience. 1999;19(13):5473-81.

7. Blakemore SJ, Choudhury S. Development of the adolescent brain: implications for executive function and social cognition. Journal of child psychology and psychiatry. 2006;47(3-4):296-312.

8. Mogedas Valladares AI, Alameda Bailén JR. Toma de decisiones en pacientes drogodependientes. 2011.

9. Verdejo-García A, Bechara A. Neuropsicología de las funciones ejecutivas. Psicothema. 2010;22(2):227-35.

10. León FG, Cruz JS, Martínez JMA, Hernández LM, Meilán JJG, Ramos JC, et al. Estudio de la toma de decisiones en una variante de la Iowa Gambling Task. REME. 2010;13(34):7.

11. Bechara A, Damasio AR, Damasio H, Anderson SW. Insensitivity to future consequences following damage to human prefrontal cortex. Cognition. 1994;50(1):7-15.

12. Smith DG, Xiao L, Bechara A. Decision making in children and adolescents: impaired Iowa Gambling Task performance in early adolescence. Developmental psychology. 2012;48(4):1180.

13. Díaz Galván KX, Ostrosky F. Desempeño neuropsicológico prefrontal en sujetos violentos de la población general. Acta de investigación psicológica. 2012;2(1):555-67.

14. Oliva A, Antolín L. Cambios en el cerebro adolescente y conductas agresivas y de asunción de riesgos. Estudios de Psicología. 2010;31(1):53-66.

15. Stevens L, Betanzos-Espinosa P, Crunelle CL, Vergara-Moragues E, Roeyers H, Lozano O, et al. Disadvantageous decision-making as a predictor of drop-out among cocaine-dependent individuals in long-term residential treatment. Frontiers in psychiatry. 2013;4.

16. Acuña I, Castillo D, Bechara A, Godoy JC. Toma de decisiones en adolescentes: rendimiento bajo diferentes condiciones de información e intoxicación alcohólica. International Journal of Psychology and Psychological Therapy. 2013;13(2):195-214.

17. Fridberg DJ, Gerst KR, Finn PR. Effects of working memory load, a history of conduct disorder, and sex on decision making in substance dependent individuals. Drug and Alcohol Dependence. 2013;133(2):654-60.

18. Malloy-Diniz LF, Neves FS, Abrantes SSC, Fuentes D, Corrêa H. Suicide behavior and neuropsychological assessment of type I bipolar patients. Journal of Affective Disorders. 2009;112(13):231-6.

19. Bridge JA, McBee-Strayer SM, Cannon EA, Sheftall AH, Reynolds B, Campo JV, et al. Impaired Decision Making in Adolescent
Suicide Attempters. Journal of the American Academy of Child \& Adolescent Psychiatry. 2012;51(4):394-403.

20. Tchanturia K, Liao P-C, Uher R, Lawrence N, Treasure J, Campbell IC. An investigation of decision making in anorexia nervosa using the Iowa Gambling Task and skin conductance measurements. Journal of the International Neuropsychological Society. 2007;13(04):635-41.

21. Herrera Giménez M. Bulimia nerviosa: emociones y toma de decisiones. Revista de Psiquiatría y Salud Mental. 2011;4(2):8895.

22. Broche-Pérez Y, Cortés-González L, Omar-Martínez E. Toma de decisiones en jóvenes con conductas antisociales: Evidencias desde el Iowa Gambling Task. Neurología Argentina. 2015;7(3):140-7.

23. Sánchez-Sarmiento P, Giraldo-Huertas JJ, Quiroz-Padilla MF. Impulsividad: una visión desde la neurociencia del comportamiento y la psicología del desarrollo. Avances en Psicología Latinoamericana. 2013;31:241-51.

24. Association AP. Manual diagnóstico y estadístico de las enfermedades mentales (DSM IV-R). Barcelona: Masson; 2002.

25. Pineda D, Kamphaus R, Mora O, Restrepo M, Puerta I, Palacio L, et al. A system of multidimensional evaluation of conduct. A scale for parents of children from 6 to 11 years old, Colombian version. Revista de neurología. 1999;28(7):672-81.

26. Swanson J, Nolan W, Pelham W. The SNAP rating scale. Resources in Education. 1982.

27. Bechara A. Iowa gambling task professional manual. Lutz: psychological assessment resources. 2007.

28. Bauer A, Timpe J, Edmonds E, Bechara A, Tranel D, Denburg NL. Myopia for the future or hypersensitivity to reward? Age-related changes in decision making on the Iowa Gambling Task. Emotion. 2013;13(1):19.

29. Coccaro EF, Sripada CS, Yanowitch RN, Phan KL. Función corticolímbica en la conducta agresiva impulsiva. Psiquiatría Biológica. 2012;19(2):46-53.

30. Duckworth AL, Kern ML. A meta-analysis of the convergent validity of self-control measures. Journal of Research in Personality. 2011;45(3):259-68.

31. Damasio AR. Descartes error: Emotion, rationality and the human brain. New York: Putnam. 1994;352:1061-70.

32. del Rocío M, Salguero P, Paíno S, Alameda JR. La hipótesis del Marcador Somático y su nivel de incidencia en el proceso de toma de decisiones. REMA. 2013;18(1):17-36. 